\title{
The Relation Between a Teacher-Based Growth Mindset Training and Minoritized Student Motivation in Mathematics
}

\author{
Christopher Seals \\ University of Illinois at Urbana-Champaign \\ Hannah Valdiviejas \\ University of Illinois at Urbana-Champaign
}

Growth mindset intervention research has centered student outcomes and has largely ignored the effects of the teacher on the student and how teachers specifically influence African American, Latinx, and Native students (AHN) in Mathematics. The current study used a pre-post experimental design to test whether a teacher-based growth mindset online intervention program influenced students' motivation (i.e., cost beliefs) on mathematics classes, particularly for AHN students. Results showed that the intervention influenced AHN students' perception of cost (i.e., effort) value. AHN status moderated outcomes on cost value beliefs, where cost value for AHN students decreased after their teacher's participation in the intervention.

Keywords: growth mindset interventions, underrepresented STEM student motivation in math, value in math, cost (effort), teacher-based intervention

\section{INTRODUCTION}

Social psychological interventions (SPI) have been intensely studied over the past two decades because of the robust long-term impact that they often have on student outcomes (Yeager \& Walton, 2011). SPIs can be particularly effective for students of color (i.e., Black, Hispanic, and/or Native students; Claro et al., 2016) for their potential to relieve psychological threat associated with race (e.g., stereotype threat). Despite positive findings, teachers' role in SPI research is understudied, especially when looking at the effects of interventions on students from racially underrepresented backgrounds in science, technology, engineering, and mathematics (STEM). In this work, we address this issue by looking at how a teacher-based SPI relates to underrepresented STEM student motivation in math classrooms.

Past research on SPIs most often provide students with the intervention; therefore, this study placed the onus on teachers as subjects who received the SPI instead. Teacher participation in SPIs is vital because the teacher has a significant role in students' overall learning process, and teacher beliefs heavily influence student outcomes (Handal, 2003; Rattan et al., 2012). Specifically, implicit theory of intelligence, or growth mindset beliefs, of teachers may directly affect students (Hattie, 2012), especially in math classrooms (Conley, 2012), where students often believe that their ability is inherent and their skills cannot increase (Jonsson et al., 2012). Moreover, teacher growth mindset beliefs may be especially influential to students who are underrepresented in math careers, as they are subject to stereotypes about their intelligence which 
assume a fixed mindset (i.e., their abilities are finite and genetically hard-wired) (Darby \& Rury, 2018; McGee, 2019). Research shows that students' adoption of a growth mindset (i.e., their brain can grow like a muscle) can often lead to greater math achievement and overall motivation (Blackwell et al., 2007). Teachers are socializers of the learning experience (Vygotsky, 1981) but are understudied in growth mindset research, which could undermine applying a student's growth mindset in a teacher's learning space (Jiang \& Rosenzweig, 2021). There is a need to study the importance of teacher inclusion in growth mindset SPI research.

The basic premise of this study is whether a professional development training centered around increasing teachers' growth mindsets has a positive relationship to students of color's motivation in math. Following expectancy-value theory (EVT; Atkinson, 1964), motivation consists of value, a person's desire and yearning to accomplish a task (Wigfield \& Eccles, 2000), and researchers note that students of color often do not value math during their adolescent years (Wang \& Degol, 2013). Of particular importance is cost value, the perceptions of how much effort is required by a task, the perceptions of valued alternative activities that one must give up completing a task, and the negative emotional and psychological consequences of doing a task (Wigfield et al., 2017). Students from African American/Black, Hispanic/LatinX, and Native (AHN; Williams, 2020) backgrounds may view math as a high-cost subject due to systemic barriers discussed in this paper. Testing student cost value in math, based on race/ethnicity, is important because it may shed light on the different social psychological processes by which growth mindset interventions alter teacher and student behavior.

Providing background on these issues, we discuss growth mindset SPIs, and especially growth mindset interventions that are teacher-based. We then discuss teacher beliefs and their effects on students. From there, we discuss student motivation, connecting growth mindset and value beliefs (i.e., cost), and how these motivational constructs may operate differently for AHN students.

\section{LITERATURE REVIEW}

\section{Growth Mindset SPIs}

The present study adds to the literature on growth mindset SPIs by testing (a) whether a teacher-based growth mindset intervention can effectively impact student motivation (i.e., cost) in math, and (b) by testing if racial identity moderates the outcome of the growth mindset intervention on student outcomes.

SPIs are exercises that use psychological principles to change students' ability beliefs and construal of challenges and could impact their sense of belonging to their community (Walton \& Cohen, 2011). This study focuses on a growth mindset intervention that comes from the implicit theory of intelligence. Implicit theory of intelligence influences students' behavior, choices, and motivation (Blackwell et al., 2007). It is a construct with two opposing views on opposite sides of a continuum in which people see and understand their intelligence or abilities (Dweck \& Leggett, 1988). For instance, on one side of the continuum is an incremental view of intelligence, also known as a growth mindset, meaning that an individual believes that their intelligence or ability can increase or is controllable. The other is an entity view of intelligence, or a fixed mindset, where a person feels that intelligence or ability is innate and uncontrollable (Eccles \& Wigfield, 2002).

During growth mindset interventions, students often receive a message about the brain being able to grow. From this perspective, intelligence is amendable (i.e., growth/incremental view) rather than fixed (i.e., entity view) (Liu, 2021). When students adopt a growth mindset, they tend to take better advantage of campus resources (e.g., tutors, counseling, and professors) and work harder (Aronson et al., 2002). With a growth mindset, students may internally attribute failure (i.e., blame themselves); however, they are equipped with a high locus of control, believing that with hard work, they can change future results (Nicholls, 1984). However, the current study leverages a teacher-oriented intervention instead of studentoriented, yet still targets student motivation in math. 


\section{Teacher Beliefs and Student Outcomes}

In the current study, as a result of completing an online growth mindset intervention, teacher beliefs are hypothesized to influence student motivation, particularly their cost beliefs in math. Teacher's beliefs about learning have a substantial impact on students' cognitive and affective learning outcomes (Zee \& Kooman, 2016). Teachers' beliefs about learning are particularly detrimental when teachers view motivation as an innate quality that students are born with (Daniels et al., 2018, p. 7), where some students are simply adequately motivated and, therefore, feel that addressing motivation is unnecessary (Shalter Bruening, 2010). Mindsets (Dweck, 2007) may provide a theoretical explanation for these beliefs. For example, teachers' pedagogical beliefs and technology value beliefs influence how teachers integrate technology in their classrooms (Ertmer et al., 2012). Teachers' beliefs about technology also influence how students learn because of explicit behaviors and more subtle messages the teacher communicates about their beliefs regarding learning and technology (Midgley et al.,1989, p. 247).

Teachers' self-efficacy also directly relates to students' motivation (e.g., Lazarides, Buchholz, \& Rubach, 2018), possibly through subconscious messages teachers transmit to their students. Therefore, there is reason to believe that teachers' beliefs towards learning abilities and growth mindset might also influence how teachers implement instruction in the classroom. Thus, the intended outcome of the intervention in our study was for teachers to embrace what they learned from the intervention about growth mindset and use that knowledge to inform the way they taught, planned their lessons, and communicated with their students. Past research supports this intended outcome because of teachers' epistemological beliefs, or conceptualization of knowledge and knowing, influence teachers' classroom behaviors, classroom instruction, and student evaluation (Prawat, 1992; Hofer \& Pintrich, 1997). Thus, supporting the inference, that teacher beliefs influence student motivation and outcomes.

Teachers' implicit theory of intelligence, or growth mindset, is a specific type of belief that may uniquely impact their students. Dweck (2010) argues that a teacher's theory of intelligence influences: how they praise students, the promotion of risk-taking, their presentation of complex activities, the labeling of students, the use of timed assignments, and if they promote challenge versus success. Highly motivating teachers tend to approach instruction by centering their students' participation and effort and prioritizing student wellbeing, positive emotions, collaboration, and building trust between them and their students (Daniels et al., 2021). Wiesman (2012) explained that both students and teachers find intrinsic motivation an important reason why students are motivated in the classroom. In their study, teachers stated their own actions and characteristics as critically responsible for students' intrinsic motivation. They found that teachers can effectively motivate their students by getting them excited about a subject or asking them their future academic plans and how they will achieve them. This finding assumes that teachers believe they can impact a student's achievement motivation by being highly efficacious for themselves and their students (Bandura, 1993 cited in Weisman, 2012). These findings promote the assertion that teachers' approach to instruction and their beliefs about learning affects students. Findings from our study may contribute to the literature in explaining the connection between teacher beliefs and how they indirectly relate to student motivation, specifically student cost beliefs in math.

\section{Growth Mindset Interventions for Teachers}

Evidence shows that many teachers feel ill-prepared to manage student motivation issues (Turner et al., 2011 cited in Daniels et al., 2021). Motivation researchers are clear that there is no single way to approach instruction to guarantee student motivation. Thus, it may be essential to help teachers understand how their approach to instruction, based on their beliefs, relates to students' motivation beliefs (Daniels et al., 2021). A prominent critique of growth mindset interventions is that they often are implemented in isolation from the students' regular instruction. Implementing interventions in isolation is problematic because this may result in the findings not being generalizable to the classroom, which undermines the practical implications of growth mindset interventions in educational contexts. Applying a growth mindset to a learning space can be especially difficult if certain instructional practices reinforce fixed mindsets in the class.

Although little literature addresses teacher interventions dealing with growth mindset, the few discussed reveal information about this effective approach to changing mindset beliefs in educational 
contexts. Zeeb et al. (2020) worked with a seventh-grade physics teacher to implement a mindset training that consisted of explicit and implicit training phases. In these implicit phases, the teacher enriched ordinary lessons with growth mindset feedback (e.g., if a student struggles to answer a question despite their effort, feedback might encourage the student to recognize the failure as an opportunity to learn). They investigated the overall outcome of this lesson-integrated training on students' beliefs and motivation. They found a positive stable training effect on students' growth mindsets, and the intervention mitigated demotivation. The findings are promising in that student growth mindset and motivation were positively affected by teacher trainings.

Another criticism of growth mindset interventions is that teachers are simply telling students to "have a growth mindset," placing the onus of shifting beliefs on the student without addressing or understanding how factors related to the teachers themselves might shape students' mindset beliefs (Liu Sun, 2018). Liu Sun (2018) conceptualized how particular teacher-student interactions might implicitly or explicitly convey fixed or growth mindset messages related to students' mathematics ability. She did this by conducting case studies of eight middle school mathematics teachers to examine differences in how they conveyed messages about mathematics ability as being a fixed or malleable trait to their students. She used a triangulation of data (i.e., observations, one-on-one interviews, and semi-structured group interviews) to understand her research question, she addressed teachers' and students' mindsets, experiences, beliefs about mathematics teaching and learning, and perceptions of how their behaviors influence student beliefs and outcomes. She found that teachers do not need to ascribe to growth-mindset beliefs to convey growth-mindset messages. For example, mathematics teachers can instruct in multidimensional ways that convey growth-mindset messages while simultaneously believing that mathematical ability is innate or fixed. Conversely, teachers can believe that mathematics ability can grow yet instruct in ways that convey more fixed-mindset messages (Liu Sun, 2018). Liu Sun's study exemplifies how the teacher's approach to instruction might be a more effective factor to focus on, rather than their beliefs and how they convey a growth or fixed mindset in the learning space. The findings promote that researchers and administrators should spend time and resources promoting instruction that conveys growth mindset beliefs.

Like in Liu Sun's study that focused on the importance of instructional practices, Seaton (2018) exemplified how teachers' responses to a brief training prompted teachers to firmly identify the training as contributing to their ability to implement adaptive motivation practices in their classrooms. In her work, she argued that teachers should benefit from instruction on mindset theory to bring about sustained change in their practices and their students' mindsets. She used a pragmatic epistemological stance with mixed methods to study teachers' responses to a mindset training. She found a statistically significant increase in growth mindsets from pre-test to post-test and at a three-month follow-up. Moreover, participants strongly identified the training as contributing to their ability to implement adaptive motivation practices in their classrooms. From the few studies mentioned, there is a consistent theme of the importance of doing growth mindset instruction rather than just believing in or having a growth mindset. Similarly, Our study will measure student motivational outcomes as an effect of teachers being taught growth mindset teaching practices from an online intervention.

Growth mindset interventions do not usually focus on teachers; however, a construct used to measure autonomy support, a closely related concept to promoting a growth mindset in students, is the Autonomy Support Intervention Program (ASIP). The ASIP is a formal intervention for teachers in the form of professional developments, addressing approaches to instruction that support student autonomy (Daniels et al., 2021). A large body of research demonstrates that students' motivation improves when teachers engage with the ASIP (e.g., Cheon et al., 2016). Daniels et al. (2021) addresses a critical gap in the literature by creating an intervention that targets both beliefs and approaches to instruction for pre-service teachers. He found that a brief, targeted, and truthful message can help increase pre-service teachers' responsibility for motivation, at least on an immediate post-test. This finding is encouraging, given that if teachers see themselves as responsible for impacting their students' motivation, they may be more likely to approach instruction more adaptively than before they received the training. Researchers know little about interventions aimed at teachers that focus on changing their approaches to instruction based on mindset 
training. We describe a teacher-based intervention study and focus on how the mindset messages relate to AHN students' perceived math value (i.e., cost).

\section{Motivation: Cost and Growth Mindset}

In this study we focus on the motivational aspect of how students are affected, particularly students' cost beliefs in math, by their teachers participating in a growth mindset intervention. Cost beliefs are the negative perceptions of engaging in a particular task (Wigfield \& Eccles, cited in Jiang \& Rosenzweig, 2021). Discussing the relationship between growth mindset and cost emphasizes the psychological processes by which a growth mindset learning experience may impact how much a student desires success or produces avoidant behaviors in a subject.

The construct of cost beliefs comes from a larger theoretical framework of motivation theory (i.e., motivation value), rooted in expectancy-value theory. In expectancy-value theory, Atkinson (1964) described complementary variables that produce an individual's behavior. Value has four components, but this study focuses on cost beliefs (effort cost), the negative consequence of engaging in the activity (Trautwein et al., 2012). Lower cost beliefs are a predictor of motivated behavior. In other words, the lower the perceived negative consequences from the effort needed to complete the task, the higher the motivation. Cost beliefs are of particular importance to motivation research. They predict intentions to leave STEM (Perez et al., 2014), attend graduate school (Robinson et al., 2019), and students' adoption of avoidance goals, negative classroom affect, procrastination, intentions to avoid studying, and exam scores in mathematics (Jiang et al., 2018). This idea can easily translate to teacher beliefs/values. Suppose a teacher promotes that understanding and succeeding in math as being "worth it" to students. In that case, this belief is more likely to be internalized by the student, possibly retaining student interest, effort, and persistence in the subject.

Motivation researchers agree that cost (a) acts as a negative force on students' motivation to learn and (b) is empirically distinct from the other components of task value (i.e., intrinsic, utility, and attainment value) (Jiang \& Rosenzweig, 2021). In their study, Jiang \& Rosenzweig (2021) examined how adolescent students' perceptions of the cost related to their intentions to pursue future activities related to a given subject, their intentions to avoid engaging with a given subject, and their expected or actual course performance in two subject areas, mathematics, and English. They found that cost was a significant predictor of both expected course grades (negatively) and avoidance intentions (positively), beyond what could be predicted by self-efficacy and task value. Cost beliefs, of which our study focuses, are related to mindset in that it strongly predicts beliefs concerning effort (Blackwell et al., 2007; Diener \& Dweck, 1978). They may also have a strong explanatory power for understanding the factors involved in student academic success, of which more popular aspects of motivation theory cannot explain alone. In other words, cost beliefs could be critical to contextualizing related constructs, like self-efficacy and interest value (Jiang \& Rosenzweig, 2021). Given how understudied (Jiang \& Rosenzweig, 2021), yet important understanding cost beliefs are to impacting students' academic achievement, we indirectly tested the relationship between growth mindset, applied by the teacher, and the cost value of students, in efforts to explain the postintervention processes. Specifically, we examine how these constructs interact with being an AHN student because cost beliefs may be particularly effective in explaining academic differences between this group and White students.

\section{Growth Mindset and Cost for AHN Students}

Only $9 \%$ of college educated AHN people in the U.S. have STEM occupations (National Research Council, 2011). Of the AHN people with STEM careers, only 6\% are career mathematics scientists (National Science Foundation, 2015), despite AHN people representing roughly $25 \%$ of the nation's population (Estrada et al., 2016). Past research has connected this STEM underrepresentation to the value beliefs of AHN students during secondary schooling age (Frenzel et al., 2010), where students' math identity is pivotal (Lee \& Anderson, 2014). This supposed lack of value (i.e., effort cost) is most likely indicative of systemic social barriers that prevent AHN students from valuing futures in STEM or math fields (Matthews, 2018). For example, urban schools, which often have many AHN students (Milner, 2006), tend 
to have lower funding and less experienced teachers (Ostrander, 2015; Vaught, 2009). Unfortunately, this often means that teachers do not have the full qualification necessary to teach math and science, making them less confident in their teaching abilities and limiting their instructional approaches to activities like rote memorization and teaching to the test (Vassallo, 2013). The under-resourcing of schools has a great deal to do with academic achievement gaps seen between AHN students and their White counterparts (Darby \& Rury, 2018). Often, marginalized students receive entirely different curriculums, despite state standards, than their counterparts (Vassallo, 2013; Darby \& Rury, 2018). These systemic differences often rob AHN students of the ability to compete with students receiving enriched instruction, to whom AHN students are compared. There may come a point where the effort involved in "swimming upstream" may become too overwhelming for AHN.

Additionally, there is a social and psychological cost (Chambers et al., 2014) to AHN students embracing academic success due to the social stigma and expectations of the White/dominant society that may not align with cultural phenomena. These social stigma and expectations involve the lack of representation of examples in subjects like math that relate to them, and the energy put into navigating a learning space where White/dominant culture is normalized (Fordham \& Ogbu, 1986). For example, Stephans et al. (2012) coined the term Cultural Mismatch Theory, which describes the psychological processes by which students who do not belong to dominant university culture (i.e., White, middle-upper class) endure when they attend university. Cultural Mismatch Theory involves students who have intersectional identities, which makes any experience complex and multifaceted. A student could be juggling the value systems of more than two groups, making their costs effort potentially higher if forced to choose between groups (Seals, 2016). School environments are similar, which also socialize the student of color in a way that endorses choices that may not be analogous to the students' cultural community's idea of educational success (Anzaldúa, 1984, p. 101). The cost effort of the student may compound because of these additional racialized factors, and it comes with psychological strain on the student (Researcher, 2016). These processes include discomfort, more challenging tasks because of cognitive load, and poor performance (Stephans, 2012). Perhaps students not belonging to dominant culture have less bandwidth to learn new concepts when dealing with the extra load prompted by cultural clashes (DuBois, 1904, p. 5) in academic spaces. This lack of bandwidth may impact how much effort they are willing to put into the academic task. These factors compound and can demotivate or increase the cost effort of math for some AHN students, or how "worth it" it is to expend limited resources on learning a math concept.

Equally demotivating and particularly impacting how teachers may approach AHN students is the rampant stereotype that AHN students are naturally, or genetically, intellectually inferior people, backed by scientific racism like biased psychological assessments (Darby \& Rury, 2018). The disproportionate number of Black students in special education programs across the U.S. (p. 122) demonstrates the idea of intellectual inferiority. Rooted in history dating back to the emancipation of African Americans from slavery, the U.S. school system has passed educational policy that promotes the assumption that Black and other marginalized students cannot do advanced work (Darby \& Rury, 2018). If educational policy reflects that assumption, then teachers may also approach AHN students under the said assumption. Teacher approaches to learning may have a further negative impact on the student if the student identifies with multiple intersecting identities of which stereotypes around their intelligence are persistent (i.e., women and African Americans; Steele, 1997). We argue that the intersectionality of, for example, these two identities, might prompt teachers to further engage with a fixed mindset about their intelligence, possibly impacting a students' cost beliefs about the task.

Because this systematic and structural racism is so ingrained in the way we educate our students in the U.S. (Ladson-Billings, 2013), the different treatment that AHN students receive systemically and, in the classroom, often goes unnoticed by teachers. In their book, Darby and Rury (2018) explain a conversation with an urban school principal in which the principal states that he could tell the future placement of a student by the end of kindergarten (p. 130). Although superficially, this looks like a comment that demonstrates how well-versed and experienced this principal might be, it also exemplifies the fixed mindset approach among educators of AHN students that conveys the notion that AHN students cannot learn. 
Perhaps AHN students pick up on these signals and messages that educators approach them with, directly impacting the effort they choose to put into subjects they are seen as incompetent to learn.

Motivational values in learning may also operate differently with AHN students. Seals (2016) argues that all four value types could benefit from a racialized lens that better aligns with AHN students than classic interpretations of motivation theory, yet keeps the same meaning and purpose. The emphasis on oppression, stereotypes, clashing cultural norms and isolation play a role when AHN students assess the cost of attempting to attain educational success (Chamber et al., 2014). Moreover, AHNs who are underrepresented in math careers may not foresee a career related to math and could conclude that math class is not useful for them (Seals, 2016). Finally, suppose AHN students do not see math success in alignment with their culturally proscribed identity group. In that case, they may not perceive math as necessary (Wigfield et al. 2004), negatively impacting how much effort they are willing to use on a specific task or subject. All these factors underscore how cost value can look different when a racialized lens is used to assess motivation. The findings from our study could speak to these factors. Endorsing a growth mindset in middle and high school math could help counter some of the effects that demotivate AHN students. As mentioned in the previous section, growth mindset beliefs can predict effort in general; however, we argue that they may especially be effective for AHN students. Past growth mindset intervention research shows academic promise for students of minoritized racial identities that face psychological threats (Yeager \& Walton, 2011; Aronson et al., 2002; Blackwell et al., 2007). These promising results could be due to growth mindset messages altering their value beliefs. Findings from this teacher-based growth mindset intervention study may help explain if students' cost effort in math differs based on racial/ethnic status, as teachers' pedagogical beliefs tend to have a more substantial positive influence on students with low prior STEM motivation than students with high motivation. (Cheng et al., 2020).

\section{Present Study}

Growth mindset interventions demonstrate effectiveness in past studies for AHN student motivation and achievement. However, a more profound exploration of these interventions might prompt practitioners to embrace them if more researchers highlight how the teacher may also influence student cost values. We hypothesized that teachers who participated in the growth mindset intervention could inspire motivation in their students by altering their students' perceived cost effort in math. As such, this study is novel because it examined the efficacy of a teacher-administered intervention, but also because it examined whether racial identity moderates the efficacy of the intervention on student cost value belief outcomes. The research questions that guided this study are listed below:

Research Questions

1. Does participation in the growth mindset intervention for teachers relate to students' cost effort value in math?

2. Does race moderate the relationship between teacher participation and student cost of the students?

\section{METHODS}

\section{Participants}

Participants were 6th - 12th-grade math teachers and their students. This study was conducted with teachers at primarily urban and suburban schools with a low population of racially underrepresented students. Our study's low percentage of AHN students reflects general U.S. population percentages, enhancing the generalizability of the students' behaviors (United States Census Bureau, 2019). Moreover, AHN students may suffer from stereotype threat (Borman et al., 2016) based on previously discussed social systemic barriers in urban and suburban settings. Additionally, we conducted this study with secondary school teachers who work with adolescents during the critical time when motivation and grades tend to decline, especially for Black and Hispanic/Latino students (Anderman, 2003). 


\section{Teachers}

The 25 (68\% female, 32\% male; 92\% White, 4\% African American, and 4\% Asian American) teachers that participated in this study (60\% middle school, $40 \%$ high school teachers) were at nine schools in one Midwestern state (44\% urban, $44 \%$ suburban, $11 \%$ rural). The number of participating teachers per school ranged from one (4\%) to eight teachers (32\%), with an average of three teachers per school. Most teachers (i.e., $\mathrm{n}=13 ; 52 \%)$ were within their first ten years of teaching, and $12(48 \%)$ taught more than ten years.

All teachers were randomly assigned to conditions, with 12 assigned to the business as usual (BAU) control condition and 13 to the mindset (intervention) condition. However, two teachers (Ms. Pumkin \& Mr. Bevinson) did not start nor complete the intervention and, consequently, were both assigned to the BAU condition group. In total, there were 11 (44\%) teachers in the mindset intervention and $14(56 \%)$ teachers in the BAU control. In support of the effectiveness of random assignment at the teacher level, there was no evidence of differences between the two conditions based on teacher's race/ethnicity $\chi^{2}(2, \mathrm{~N}=25)$ $=2.06, p<.36$, sex/gender $\chi^{2}(1, \mathrm{~N}=25)=2.02, p=.65$, teacher's school $\chi^{2}(8, \mathrm{~N}=25)=12.52, p=.13$, and the amount of years teaching (greater or less than 10 years) $\chi^{2}(1, \mathrm{~N}=25)=1.07, p=.30$.

\section{Students}

The study consisted of 1,653 participating students. In total, $844(51 \%)$ of the students were male, while $794(48 \%)$ were female, with less than $1 \%$ identifying as non-binary gender. Participants were ethnically and racially diverse, with 224 (14\%) identified as Black, 126 (8\%) Asian, 120 (7\%) Latinx, 134 (8\%) multiracial, and 987 (60\%) White. We purposefully measured race/ethnicity as binary, splitting students into two groups a) AHN and multiethnic students $(n=517)$ and $b)$ White and Asian students $(n=1,113)$. Due to AHN students often making up such a small percentage of public schools' student body, we included all AHN, non-White and non-Asian students in one group. Collapsing these demographic groups helped us achieve enough power for a quantitative comparison. White and Asian students were included in the second group because White students do not face comparable social and systemic barriers that AHN students face, as previously discussed. Moreover, Asian students were placed in the second group with White students because Asian students are often seen as model minorities (e.g., Wu, 2017) and do not face the same pressure and expectations of failure in U.S. STEM settings. We understand that this decision to break race down into two groups is convoluted. However, we also saw this as an opportunity to tell a unique story about motivation among AHN students.

In total, $546(33 \%)$ students also identified as being in the free and reduced lunch government program. For the sake of this study, we defined an AHN or minoritized student as any student who does not racially or ethnically identify with being White or Asian. Table 1 summarizes student demographic information by condition.

\section{TABLE 1 STUDENT DEMOGRAPHICS BY CONDITION}

\begin{tabular}{llll}
\hline & Student & & \\
\cline { 3 - 4 } $\mathrm{N}$ & Mindset & BAU & Total \\
\hline Sex & 303 & 541 & 844 \\
Male & 312 & 482 & 794 \\
Female & & & \\
Race/Ethnicity & 78 & 146 & 224 \\
\hline Black & 38 & 88 & 126 \\
Asian & 38 & 82 & 120 \\
Latinx & 22 & 17 & 39 \\
Various non-White & 390 & 597 & 987 \\
White & & & \\
\hline
\end{tabular}




\begin{tabular}{|c|c|c|c|}
\hline Multi-Racial & 49 & 85 & 13 \\
\hline \multicolumn{4}{|l|}{ Race Binary } \\
\hline White \& Asian & 428 & 685 & 1,113 \\
\hline AHN \& Multi & 187 & 330 & 517 \\
\hline \multicolumn{4}{|l|}{ School } \\
\hline FHS (2) & 0 & 85 & 85 \\
\hline IJHS (8) & 284 & 560 & 844 \\
\hline IMS (1) & 0 & 51 & 51 \\
\hline MCA (3) & 0 & 84 & 84 \\
\hline PHS (5) & 117 & 177 & 294 \\
\hline SCMS (1) & 0 & 73 & 73 \\
\hline MMMS (2) & 94 & 0 & 94 \\
\hline IHS (2) & 99 & 0 & 99 \\
\hline NHS (1) & 29 & 0 & 29 \\
\hline \multicolumn{4}{|l|}{ Grade Level } \\
\hline $6^{\text {th }}$ & 96 & 140 & 236 \\
\hline $7^{\text {th }}$ & 83 & 383 & 466 \\
\hline $8^{\text {th }}$ & 200 & 244 & 444 \\
\hline $9^{\text {th }}$ & 103 & 63 & 166 \\
\hline $10^{\text {th }}$ & 92 & 45 & 137 \\
\hline $11^{\text {th }}$ & 12 & 89 & 101 \\
\hline $12^{\text {th }}$ & 37 & 59 & 96 \\
\hline \multicolumn{4}{|l|}{ Free Lunch } \\
\hline Yes & 192 & 354 & 546 \\
\hline No & 418 & 647 & 1065 \\
\hline
\end{tabular}

Note: $\mathrm{BAU}=$ Business as usual control condition. AHN = African American/Black, LatinX/Hispanic, and Native American. Value in parenthesis, next to school, is the number of participating teachers in that school.

There was no evidence of difference between the two conditions based on students' sex/gender $\chi^{2}(2, \mathrm{~N}$ $=1,638)=3.71, p=.16$ or students' free and reduced lunch status $\chi^{2}(1, \mathrm{~N}=1,170)=2.56, p=.11$. However, there was evidence of difference between condition based on race/ethnicity $\chi^{2}(5, \mathrm{~N}=1,630)=12.96, p<$ .02 , and grade level, $\chi^{2}(6, \mathrm{~N}=1,646)=210.43, p<.00$. There were fewer total African American and Hispanic students in the mindset condition for race, but only minor differences in percentage representation between conditions. Moreover, when race was aggregated into two groups (i.e., 517 AHN students vs. 1,113 White/Asian), there was no evidence of difference between condition based on race/ethnicity $\chi^{2}(1, \mathrm{~N}=$ $1,630)=.78, p<.38$. Finally, because school and grade were dependent on the random assignment of the teacher, differences between conditions were expected and were statistically controlled in all models. This test indicated an equivalent distribution of students in the BAU and mindset conditions across gender, free lunch status, and AHN status.

Most importantly, we automatically assigned students to conditions based on their math teacher's random assignment. Therefore, the number of students per school and grade was dependent on their teacher. See Table 2 for the number of students per teacher, separated by condition. There were $624(38 \%)$ students in the mindset condition and 1,029 (62\%) in the BAU condition. 
TABLE 2

STUDENTS BY TEACHERS BY CONDITION

\begin{tabular}{|c|c|c|c|}
\hline School & Teacher & Mindset & BAU \\
\hline \multicolumn{4}{|l|}{$\overline{\text { SCMS }}^{\mathbf{r}}$} \\
\hline & Ms. Reed & - & $73(7 \%)$ \\
\hline \multicolumn{4}{|l|}{$\mathbf{I J H S}^{\mathrm{s}}$} \\
\hline & Ms. Buffer & - & $138(13 \%)$ \\
\hline & Ms. Knowles & - & $129(13 \%)$ \\
\hline & Ms. Love & - & $136(13 \%)$ \\
\hline & Mr. Grayson & - & $134(13 \%)$ \\
\hline & Ms. Weigh & $84(13 \%)$ & - \\
\hline & Ms. Royce & $72(12 \%)$ & - \\
\hline & Ms. Wolfe & $128(21 \%)$ & - \\
\hline & Mr. Bevinson* & - & $22(2 \%)$ \\
\hline \multicolumn{4}{|l|}{$\mathbf{I M S}^{\mathbf{u}}$} \\
\hline & Ms. Taylor & - & $51(5 \%)$ \\
\hline \multicolumn{4}{|l|}{ FHS $^{\mathbf{u}}$} \\
\hline & Ms. Rose & - & $22(2 \%)$ \\
\hline \multirow{2}{*}{\multicolumn{4}{|c|}{$\mathbf{P H S}^{\mathrm{s}}$}} \\
\hline & & & \\
\hline & Ms. Quick & - & $94(9 \%)$ \\
\hline & Mr. Viola & - & $83(8 \%)$ \\
\hline & Ms. Hukstable & $17(3 \%)$ & - \\
\hline & Mr. Mills & $19(3 \%)$ & - \\
\hline & Ms. McMahon & $81(13 \%)$ & - \\
\hline \multicolumn{4}{|l|}{ MCA $^{u}$} \\
\hline & Ms. Olds & - & $17(2 \%)$ \\
\hline & Ms. Pumkin* & - & $30(3 \%)$ \\
\hline & Mr. Chan & - & $37(4 \%)$ \\
\hline \multicolumn{4}{|l|}{ MNHS $^{\mathbf{u}}$} \\
\hline & Ms. Simpson & $29(5 \%)$ & - \\
\hline \multicolumn{4}{|l|}{ IHS $^{\text {s }}$} \\
\hline & Mr. Carter & $55(8 \%)$ & - \\
\hline & Mr. Nelson & $45(7 \%)$ & - \\
\hline \multirow{4}{*}{ MMMS $^{\mathrm{s}}$} & & & \\
\hline & Ms. Fey & $44(7 \%)$ & - \\
\hline & Ms. Poller & $50(8 \%)$ & - \\
\hline & Total & $624(38 \%)$ & $1029(62 \%)$ \\
\hline
\end{tabular}

Note: * Teacher originally assigned to mindset intervention (experimental) condition but did not complete nor start the intervention. BAU $=$ Business as usual control condition. MS $=$ Mindset intervention condition.

r. School classified as rural school.

s. School classified as suburban school.

u. School classified as urban school.

\section{Teacher Intervention Program}

This study used a waitlist-control comparison of the participating teachers. At the beginning of the school year, we randomly assigned half of the participating teachers to complete the online mindset 
intervention in late September. We gave the other half access to complete the mindset intervention after the study concluded. The waitlisted business as usual (BAU) control group teachers were not required to complete any training for the study. However, they were aware that their counterparts participated in a growth mindset intervention.

The mindset treatment group completed the online Mindsetworks® Mindsetmaker teacher professional development program, of which no other study, to our knowledge, has used for teacher-based interventions. Mindsetworks ${ }^{\circledR}$ is an award-winning interactive online program that provides teachers, students, and parents with a growth mindset-driven learning and teaching approach. The content of the Mindsetmaker program modeled the intervention content of two previous studies: Blackwell et al.'s (2007) study and the online module used in Paunesku et al.'s (2015) study. Both interventions covered research that argues how intelligence and abilities strengthen with practice. Researchers that use the Mindsetworks ${ }^{\circledR}$ Brainology program use similar content, which is made for students and not teachers. (e.g., Schmidt et al., 2017).

The Mindsetmaker program is specifically an online professional development course that teaches educators, individually, about growth mindset while also providing tools, resources, and teaching strategies for implementing a growth mindset culture in their classrooms and schools. Mindsetmaker is based on research that supports that teacher math beliefs (Handal, 2003; Stipek et al., 2001), teacher implicit views of intelligence/mindset (Rattan et al., 2012), and feedback from teachers (Mueller \& Dweck, 1998) influences student performance. Mindsetmaker, as an online multimedia course, was built to fit the needs of busy educators by being easily accessible online, providing tools/tips that can be used in the classroom.

The lessons are broken into five parts so that educators can learn the fundamentals of growth mindset research. The five parts include one introduction module plus four complete modules. Each module has a video lesson about relevant research on growth versus fixed mindset and helps teachers explore related classroom practices influenced by implicit theories of intelligence. There was also an online discussion forum where teachers shared successes, adversities, and asked questions to other teachers who were in the experimental group. Moreover, there were online assessments for teachers to learn about their own mindset beliefs.

The introduction module overviews mindset (implicit theory of intelligence) and the motivation courses. Module 1 introduced growth and fixed mindsets, explained how mindset drives effort and performance and discussed ways to assess student performance. Module 2 discussed how teachers' communication with students could affect student mindsets and shared language and practices reinforcing a growth mindset. Module 3 discussed research that described teaching practices that promote a growth mindset. Last, Module 4 discussed how endorsing a growth mindset improves student motivation, achievement, and behavior. Module 4 also taught educators how to teach growth mindset study habits. Each session took about 10 to 30 minutes, and we asked teachers to complete the four modules across 30 days from September to October. The time between each module allowed teachers to reflect on and use the material in their classroom practice.

\section{Sampling Procedure}

We recruited the 25 participating teachers by word of mouth, email, and social media. The teachers completed the Mindsetmaker intervention program from September to October (Time 1) toward the beginning of the school year.

In October (Time 1), the students completed a survey that measured motivation dynamics. The motivation scales included (a) student's view of ability and (b) the value of math. We asked students to complete this survey again in December (Time 2). They completed the surveys on devices with online access.

We compensated the math teachers with $\$ 50$ personal gift cards for the time they gave in their classroom for this study and data collection. We did not compensate the students. We were granted exemption through our institution's Institutional review board according to \#i054491 at Michigan State University. 


\section{Measures and Covariates}

We collected the data two times, Time 1: September/October \& Time 2: December, over the 15-week fall semester. The lead researcher met with the teachers several times throughout the semester, including before completing the intervention, after completing the intervention, and administering the surveys to their students. The teachers and the researcher administered the questionnaires to the students during their regularly scheduled math courses. The surveys for both teachers and students presented each item with Likert scale response choices. We report the reliability (Cronbach's alpha) of each student's measured construct in Table 3.

TABLE 3

RELIABILITY OF THE CONSTRUCT/SCALE

\begin{tabular}{lll}
\hline & Time 1 & Time 2 \\
\hline View of ability: Mindset (6) & & \\
& .833 & .851 \\
Effort cost (5) & .914 & .940 \\
\hline
\end{tabular}

Note: Each Dependent Variable in the study with number of items in parenthesis.

\section{Student Measures}

Implicit View of Ability/Intelligence or Mindset. At Time 1 and Time 2, students' beliefs about their math intelligence's plasticity were measured $(\square \mathrm{T} 1=.83 ; \square \mathrm{T} 2=.85)$. The measure included three incremental items and three entity theory items (Dweck, 2007) and served as a fidelity check for mindset after teacher participation in the intervention. The students answered items on a seven-point Likert scale from strongly disagree to strongly agree.

Task Values and Effort Cost. At Time 1 and Time 2, we issued effort cost (five items; $\square \mathrm{T} 1=.91$; $\square \mathrm{T} 2=.94)$ at both time points and the scale was adapted from the procedure of Flake et al. (2015). The students answered all except one on a seven-point Likert scale from strongly disagree to strongly agree. The five Likert-type questions were: a) This class demands too much of my time, b) I have to put too much energy into this class, c) This class takes up too much time, d) This class is too much work, and e) This class requires too much effort.

\section{Data Analysis}

Preliminary analyses consisted of missing data analysis and examining each dependent variable's descriptive statistics and bivariate correlations. We hypothesized that students with teachers in the mindset condition would show a significant reduction in their cost beliefs of math from Time 1 to Time 2 (RQ1) and that race would be a moderating factor that explained the significant reduction in students' cost beliefs of math from Time 1 to Time 2 (RQ2).

\section{Primary Analyses}

We used a 3-level (teacher, student, observation) hierarchical linear model (HLM or multilevel model; West et al., 2014, Chapter 7) for both research questions. We conducted a separate HLM for every dependent variable using the teacher's condition assignment as the predictor variable. The critical test of the mindset intervention was the two-way interaction between Time and Intervention. This analytical approach accounts for the lack of independence among repeated measures nested within students and students nested within the same class (teacher). To answer research question 2, we added a three-way interaction (race $\mathrm{X}$ time $\mathrm{X}$ intervention) to each model, with race working as a moderating variable. The covariates in all HLM models included race/ethnicity, sex, school, grade level, and free or reduced lunch status. Moreover, we used SAS University Edition software to conduct the analysis. 


\section{RESULTS}

\section{Preliminary Analyses}

Missing Data Analyses

Of the 1,653 total participating students, 1,548 (94\%) of the students completed the Time 1 survey, and 1,300 (77\%) completed the survey at Time 2, while only 1,195 (72\%) students participated at both time points. The sampling procedure for this study allowed participants to be included in the analysis if they completed at least one survey.

We used Little's MCAR test (Little \& Rubin, 2002) to assess whether missingness was significantly related to dependent variables and predictors. Missing data ranged from 0 and 3\% across all survey items and demographic data, and Little's MCAR test was not statistically significant at Time $1, \chi^{2}(10)=14.04$, $p=.171$, or at Time $2, \chi^{2}(10)=13.27, p=.209$. This finding strengthens confidence that missing data were missing completely at random (MCAR).

\section{Bivariate Correlations and Descriptive Statistics}

To examine the convergent and discriminant validity of the motivational outcomes collected, we examined bivariate correlations for both Time 1 and 2. Correlations among the variables were significant and consistent with prior research for student responses: Growth mindset negatively correlated with cost at both Time $1(-.21)$ and Time $2(-.25)$. This result means that growth mindset and cost were not strongly related. Moreover, growth mindset data at Time 1 was strongly correlated to growth mindset Time 2 data (.61), and cost data at Time 1 was strongly correlated to cost Time 2 data (.60). This result indicates that our students were consistent in their responses.

We also examined the means and standard deviations for cost. In Table 4, cost value slightly increased from Time 1 to Time 2 for both students in the control group and mindset condition group. This infers that learning math became more of a burden over time and had a higher psychological cost to all students.

\section{TABLE 4 \\ DESCRIPTIVE STATISTICS FOR STUDENTS}

\begin{tabular}{|c|c|c|c|c|}
\hline Group & Mindset & & BAU & \\
\hline \multirow[t]{3}{*}{ Time } & 1 & 2 & 1 & 2 \\
\hline & $\mathrm{N}=577$ & $\mathrm{~N}=460$ & $\mathrm{~N}=971$ & $\mathrm{~N}=840$ \\
\hline & $\mathrm{M}(\mathrm{SD})$ & $\mathrm{M}(\mathrm{SD})$ & $\mathrm{M}(\mathrm{SD})$ & $\mathrm{M}(\mathrm{SD})$ \\
\hline Cost & $3.50(1.63)$ & $3.53(1.76)$ & $3.42(1.59)$ & $3.63(1.63)$ \\
\hline
\end{tabular}

Note . Cost $=$ effort cost value. BAU $=$ Business as usual control group.

\section{Primary Analyses}

Research Question 1

The analysis showed no significant change for students' cost beliefs. The descriptive data shows a slight increase in cost for students in both the mindset and control groups, but the changes were not significant. These findings infer that the teacher intervention did not influence the cost value of the students, despite their condition. In Table 5 we demonstrate that from Time 1 to Time 2 there was not a significant change, $\beta=-.16, S E=.09, p=.05$ (i.e., time $\mathrm{x}$ intervention, $\beta 16$ ). 


\section{TABLE 5}

COVARIATE ANALYSIS: TEACHER MINDSET INTERVENTION ON STUDENT COST (OF EFFORT) IN MATH

\begin{tabular}{|c|c|c|}
\hline \multirow[b]{2}{*}{ Model $^{\mathrm{a}}$} & \multicolumn{2}{|c|}{ Cost } \\
\hline & 1 & 2 \\
\hline Omnibus $^{\mathrm{b}}$ & $519.09 * *$ & $518.73 * *$ \\
\hline \multicolumn{3}{|l|}{ Fixed effects } \\
\hline Intercept, $\beta 0$ & $3.79 * *(.26)$ & $3.77 *(.26)$ \\
\hline Time, $\beta 1$ & $.19 * *(.05)$ & $.19 * *(.05)$ \\
\hline Intervention, $\beta 2$ & $.16(.32)$ & $.16(.32)$ \\
\hline Race, $\beta 3$ & $.16(.08)$ & $.20 *(.09)$ \\
\hline Gender, $\beta 4$ & $.05(.07)$ & $.05(.07)$ \\
\hline \multicolumn{3}{|l|}{ School, $\beta 5$} \\
\hline FHS, $\beta 6$ & $.39(.52)$ & $.38(.52)$ \\
\hline IHS, $\beta 7$ & $-.53(.50)$ & $-.54(.50)$ \\
\hline IMS, $\beta 8$ & $-1.08(.61)$ & $-1.08(.61)$ \\
\hline MCA, $\beta 9$ & $-.56(.42)$ & $-.57(.42)$ \\
\hline NHS, $\beta 10$ & $-.63(.68)$ & $-.59(.68)$ \\
\hline PHS, $\beta 11$ & $-.09(.38)$ & $-.09(.38)$ \\
\hline SCMS, $\beta 12$ & $-.77(.60)$ & $-.77(.60)$ \\
\hline MMMS, $\beta 13$ & $-.93(.50)$ & $-.95(.50)$ \\
\hline Free Lunch, $\beta 14$ & $-.17 *(.09)$ & $-.18 *(.09)$ \\
\hline Grade Level, $\beta 15$ & $-.09(.07)$ & $-.09(.07)$ \\
\hline Time $\mathrm{X}$ Intervention, $\beta 16$ & $-.16(.09)$ & $-.09(.10)$ \\
\hline Time $X$ Intervention $X$ Race, $\beta 17$ & & $-.30 *(.15)$ \\
\hline \multicolumn{3}{|l|}{ Random effects } \\
\hline Teacher & $.27 *(.12)$ & $.27 *(.12)$ \\
\hline Student & $1.40 * *(.08)$ & $1.40 * *(.08)$ \\
\hline Residual & $1.08 * *(.04)$ & $1.08 * *(.04)$ \\
\hline$-2 \mathrm{LL}$ & 9879 & 9877 \\
\hline
\end{tabular}

We found no significant difference in student cost beliefs when measuring all students, contrary to our hypothesis. This finding alone leads us to assume that teachers in the growth mindset intervention may not have made any changes to their teaching practices after participating in the growth mindset intervention.

\section{Research Question 2}

RQ2 looked at the differential outcomes of the intervention on student motivation based on race. Contrary to the results from question 1, these results showed a significant decrease in cost beliefs for AHN students whose teachers participated in the mindset intervention from Time 1 to Time $2, \beta=-.30$, S.E. = $.15, \mathrm{p}=.05$ (see Table 5). Complete findings for RQ2 are in Table 5 (time $\mathrm{x}$ intervention $\mathrm{x}$ race, $\beta 17$ ). These findings suggest that racial status (i.e., $\mathrm{AHN}$ ) is a moderating variable between the effects of the intervention on students' cost value. AHN students did view math as less of a burden over time, but only for those AHN students whose teacher participated in the intervention. AHN students in the control group 
and non-AHN students whose teacher participated in the intervention had increased cost beliefs over time, like most student participants.

See Figure 1 and Figure 2 for an illustration of the changes over time. We measured four groups (control $\mathrm{AHN}$, control non-AHN, mindset intervention AHN, mindset intervention non-AHN), and three of the four groups increased in cost beliefs from October to December. This finding shows that, over time, most students view learning math as more of a burden-however, one of the four groups had an opposite view. Figure 2 best illustrates how AHN students' (whose teacher participated in the intervention) cost beliefs did not increase over time but instead decreased, corroborating with our hypothesis.

\section{FIGURE 1}

\section{AHN STATUS MODERATES EFFECTS OF COST (EFFORT) VALUE}
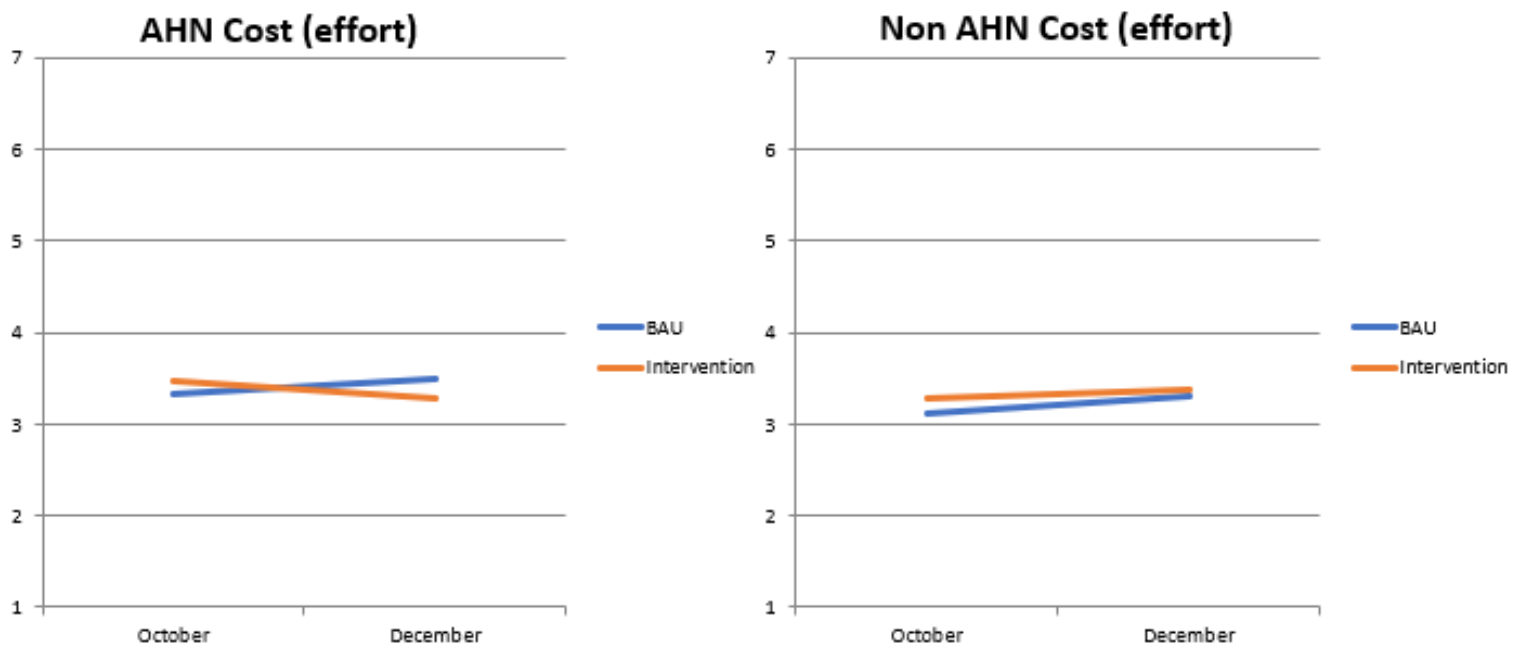

Note. The Y-axis indicates mean score on the cost scale.

FIGURE 2

AHN STATUS MODERATES EFFECTS OF COST (EFFORT) VALUE (ZOOMED IN)

AHN Cost (effort)

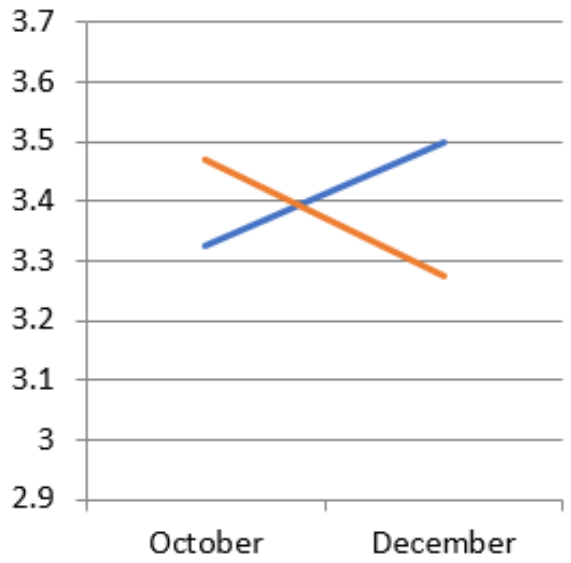

Non AHN Cost (effort)

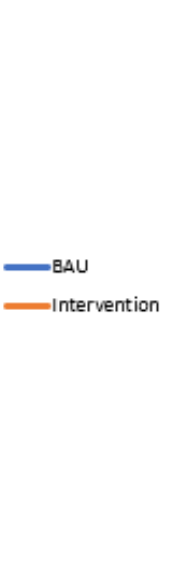

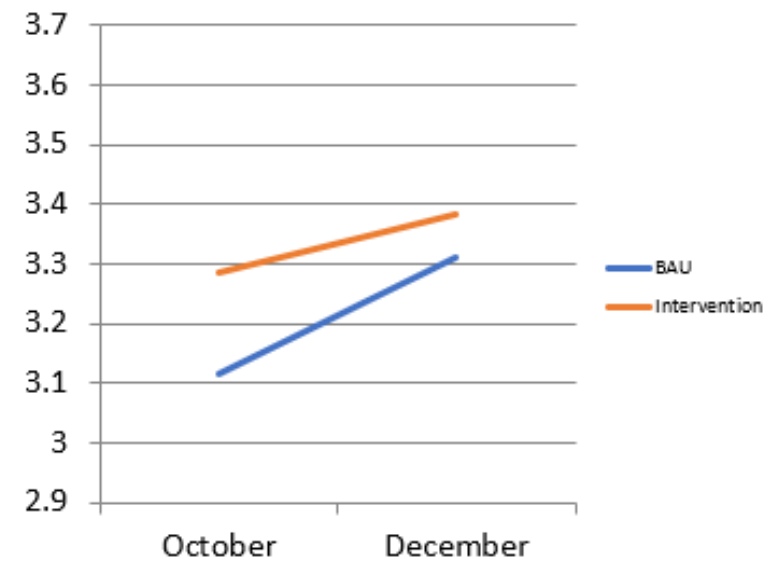

Note. The Y-axis indicates mean score on the cost scale. 


\section{Summary of Results}

Most students in teachers' classrooms who did the growth mindset intervention had an increase in cost beliefs. However, students' racial status as, AHN, served as a moderating variable, illustrating a significant decrease in cost value beliefs overtime, for AHN students in the intervention group. This finding implies that the growth mindset intervention noticeably impacted the teachers' behavior in some way, which impacted AHN students' cost beliefs about math. Therefore, overall findings indicate that the teacher-based intervention showed measurable differences in students in the intervention group, but only for AHN students.

\section{DISCUSSION}

We originally found that students' cost beliefs about learning math were not related to whether their teacher participated in the mindset condition; however, when stratified by race, AHN students were significantly affected by their teachers' participation in this growth mindset intervention. This finding is interesting because students can only be influenced by the intervention if there was a change in their teacher's behavior, which was not measured directly in this study. Although we did not explicitly test teachers' beliefs, the difference in students' beliefs based on whether the teacher participated in the intervention should be viewed as a proxy to a change in teachers' instructional approach. We specifically did not analyze whether the professional development significantly impacted the teachers' beliefs because we were most interested in the intervention's impact on students. If the intervention had an impact on teachers, but the teachers did not in some way adjust their approach to instruction, then there should be no differences in student outcomes. The point of this intervention was to impact the students' cost value beliefs. Therefore, we analyzed the directly pertinent variable to us, students' cost beliefs. We will discuss the measured motivational construct finding to shed light on the psychological processes by which the growth mindset intervention and teachers' practices altered students' motivation.

\section{Differential Outcomes of Intervention Based on the Race/Ethnicity of the Students}

Research question 2 showed that the cost (effort) value of math learning for AHN students had a significant interaction with teachers' growth mindset intervention and time (Table 5), meaning that racial status is a moderating variable for the effects on cost value. This finding implies that a growth mindset classroom, or a classroom in which the teacher implements elements of a growth mindset instruction, endorses AHN students to see math success as less of an expense or burden. As previously discussed, growth mindset intervention literature argues that students who face psychological or stereotype threat due to their underrepresented identity group use the growth mindset messages to protect their self-image and relieve themselves of threat or burden (Yeager \& Walton, 2011). This relief of threat not only allows students the freedom to enjoy math, but it particularly allows AHN students to view the threat or burden of math success as less costly over time.

Praising students for their effort instead of their outcome, promoting risk-taking, giving students more autonomy, or being more cognizant of the use of student labels are a few examples of how the math teachers in the treatment group may have relieved their students from the common fears of math and sparked enthusiasm instead. Although we did not test this in the current study, the intervention given to the teachers improved the cost beliefs of the AHN students, and highly motivated teachers tend to approach instruction in these ways (Daniels et al., 2021). Therefore, we imply that teachers may have employed these types of changes in their instruction that would cause AHN students' cost beliefs to improve. Students in past research who faced psychological threat in math used the messages about growth mindset to protect their self-image (Sherman \& Hartson, 2011) when approaching math and, therefore, did not perceive math as threatening, burdensome, or costly. A growth mindset endorsed learning space created by the teacher perhaps relieved the students of the fear of math, allowing them to enjoy it genuinely. This idea would be especially true for math which is often viewed as complicated or a subject that requires innate abilities for success (Jonsson et al., 2012). 
This unique finding showed that racially minoritized status plays a role in the relationship between growth mindset and cost value. This finding also encourages racialized cost to be considered in the study of cost beliefs because daily decisions influenced by a student's perception of cost value can be rooted in more profound racial phenomena. For example, being the only one of a particular race in an honors math course may impact a student's sense of belonging and wellbeing, impacting their cost value (Seals, 2016). When considering cost beliefs of an AHN student, the values of their culture may clash with the values of the White normed education system. A clash in values between two systems (i.e., school, community, family) may put the student in a position where they must choose which value system to ascribe. Making a choice, however, means that the student may be abandoning the competing value system. Leaving one value system behind comes with psychological consequences, meaning a student could be juggling the value systems of two or more groups, which could make the costs potentially higher if forced to choose between groups (Chambers, 2014; Seals, 2016).

Juggling two or more value systems (Anzaldua, 1987, p. 60) may help contextualize our findings by considering that although AHN students might flourish in their math class, they may also be navigating whether it is worth the effort to succumb to a "math" identity. Yeskel (2008) explained this conflict in terms of socioeconomic class and predominantly White institutions. His explanation is transferable and can also be used to explain AHN identities in predominantly White subject areas. He explains that if a student is from a low-income background, they are often presumed to bring values that differ and conflict with higher education. In contrast, middle-high income background students are often rewarded because their values tend to align with the teachers' values (most often White, middle-class women) and education institutional values (Vassallo, 2013). For example, universities in the U.S. value and promote independence, a value that feels normal to affluent students who have been socialized in similar ways. However, low-income students, for example, are often socialized to value interdependence within their families and communities, out of necessity and culture. This cultural difference in upbringing may prompt a sort of cultural mismatch for low-income students entering higher education spaces (Stephans et al., 2012). This mismatch could create a psychological conflict regarding whether a student thinks predominantly White STEM subjects (McGee, 2020) like math are worth the effort (cost value) of going through this inner turmoil in a subject that is not only made up of primarily White people but is also structured in a way that caters to White comprehension and comfort (Stovall, 2006).

We argue that during middle and high school, students may feel that they must choose whether to proceed and go through the conflict of adopting math as part of their identity. This choice could mean putting one's home values aside, affecting the cost value, or racialized opportunity cost students place on math (Chambers et al., 2014). The results of this teacher-based growth mindset intervention address this issue and show a positive impact to cost value of AHN students in a math learning context.

Finally, we must also consider that AHN students may not feel that math is worth their effort because of the more tangible systematic barriers, often related to socioeconomic status, that block them from achieving a successful future in math (U.S. Department of Education, 2005; Estrada et al., 2016). Therefore, putting time and effort into something that will not return the investment might feel like a waste of time.

These findings are promising; this intervention was easy to administer and did not take much time from the teachers' work week. Given the low maintenance of this intervention, these significant effects could greatly improve mathematics instruction and, therefore, issues of retention and representation in STEM majors and STEM careers of AHN students. This intervention might also be suitable across developmental trajectories and could be given to teachers of higher and lower grades than we tested.

\section{Limitations \& Delimitations}

The primary limitation of the present study is that we did not measure teacher outcomes, ask teachers for examples of lesson plans, nor inquire about their pedagogical beliefs. These data could have provided information, which could contextualize teachers' approach to the classroom and their application of a growth mindset. These teacher materials could have helped us to understand better and nuance teacher beliefs. This information also could have served as a measure of the effectiveness of the mindset intervention. This study, however, included a group of math teachers at various schools, in different settings 
(e.g., urban, suburban, rural), in neighboring school districts, and both high school and middle schools. The geographical diversity of the teachers infers that teachers who adopt various pedagogical philosophies were represented in this study. However, the diversity of teachers does not guarantee nor identify which teachers have varying pedagogical beliefs. In future studies, qualitative methods should be considered to best address this limitation.

A second limitation dealing with the teachers includes the way the participating teachers were selected. We recruited teachers for this study on a volunteer basis. Randomly assigning the teachers into conditions assuaged the concern of the comparability of participating teachers; but the study was still limited to teachers who expressed interest in the and therefore may represent a particularly driven or engaged group.

The lack of robust statistical power when analyzing samples of minoritized populations was also a limitation in this study. One of the main issues with working with minoritized populations is that the sample size is smaller than the overall sample size, lowering the statistical power it can produce. The sample size issue can change most interpretations of the findings in the research. Future research could be done in a setting where minoritized students are the numerical majority in that particular space, such as a school with a large population of AHN students. However, for the current research, we were interested in a school setting that represented the national numbers of the underrepresentation in STEM, leaving us to a smaller group of students.

\section{Future Research}

The limitations section of this study suggests several items to consider in future research. One item to consider is to add a qualitative component that can contextualize and nuance the teachers' beliefs. An additional quantitative component that measures participating teachers' application of growth mindset practices would also be beneficial. Finally, future studies should have more teachers as participants, since randomization occurred at the teacher level.

\section{Implications}

Findings from this study illustrate the important role of teachers and, in particular, teacher beliefs and practices from the outcomes of growth mindset interventions on student motivation and achievement. Most importantly, from the findings of this study, we advocate for the inclusion of racialized cost to be considered in research of cost beliefs and motivation because AHN student motivation may be largely rooted in more profound racial phenomena. Moreover, findings from this study could inform teacher education and teacher professional development, particularly for motivating AHN students in math subjects. From the results of this study, we encourage teachers and school administrators to invest their resources in adopting a growth mindset to shift the teacher practices and the learning culture of their schools as it pertains to teachers' beliefs of AHN students' intelligence. An intervention in the form of professional development for teachers is an efficient approach for many schools, considering that access to teachers is more manageable than accessing the entire student body. We foresee this intervention being suitable across ages and educational contexts due to its facility in implementation and its focus on teachers rather than students.

\section{REFERENCES}

Anderman, L.H. (2003). Academic and social perceptions as predictors of change in middle school students' sense of school belonging. The Journal of Experimental Education, 72, 5-22. https://doi.org/10.1080/00220970309600877

Anzaldúa, G. (1987). Borderlands: The new mestiza/La frontera (3rd ed.). Aunt Lute Books.

Aronson, J., Fried, C., \& Good, C. (2002). Reducing the effects of stereotype threat on African American college students by shaping theories of intelligence. Journal of Experimental Social Psychology, 38, 113-125. https://doi.org/10.1006/jesp.2001.1491

Atkinson, J.W. (1964). An introduction to motivation. Princeton, N.J.: Van Nostrand. 
Blackwell, L.S., Trzesniewski, K.H., \& Dweck, C.S. (2007). Implicit theories of intelligence predict achievement across an adolescent transition: A longitudinal study and an intervention. Child Development, 78(1), 246-263. https://doi.org/10.1111/j.1467-8624.2007.00995.x

Borman, G.D., Grigg, J., \& Hanselman, P. (2016). An effort to close achievement gaps at scale through self-affirmation. Educational Evaluation and Policy Analysis, 38(1), 21-42. https://doi.org/10.3102/0162373715581709

Bronson, P. (2007). How not to talk to your kids. New York.

Chambers, T.V., Huggins, K.S., Locke, L.A., \& Fowler, R.M. (2014). Between a "ROC" and a school place: The role of racial opportunity cost in the educational experiences of academically successful students of color. Educational Studies, 50(5), 464-497. https://doi.org/10.1080/00131946.2014.943891

Cheng, L., Antonenko, P.D., Ritzhaupt, A.D., Dawson, K., Miller, D., MacFadden, B.J., . . Ziegler, M. (2020). Exploring the influence of teachers' beliefs and 3D printing integrated STEM instruction on students' STEM motivation. Computers \& Education, 158. https://doi.org/10.1016/j.compedu.2020.103983

Cheon, S.H., Reeve, J., \& Song, Y.G. (2016). A teacher-focused intervention to decrease P.E. students' motivation by increasing need satisfaction and decreasing need frustration. Journal of Sport and Exercise Psychology, 38(3), 217-235. https://doi.org/10.1123/jsep.2015-0236

Claro, S., Paunesku, D., \& Dweck, C.S. (2016). Growth mindset tempers the effects of poverty on academic achievement. Proceedings of the National Academy of Sciences, 113(31), 8664-8668. https://doi.org/10.1073/pnas.1608207113

Conley, A.M. (2012). Patterns of motivation beliefs: Combining achievement goal and expectancy-value perspectives. Journal of Educational Psychology, 104, 32-47. https://doi.org/10.1037/a0026042

Daniels, L.M., Goegan, L.D., Radil, A.I., \& Dueck, B.S. (2021). Supporting pre-service teachers' motivation beliefs and approaches to instruction through an online intervention. British Journal of Educational Psychology, 91(2), 775-791. https://doi.org/10.1111/bjep.12393

Daniels, L.M., Poth, C.A., \& Goegan, L.D. (2018). Enhancing our understanding of teachers' personal responsibility for student motivation: Mixed insights informing theory, measurement, and practice. Frontiers: Educational Psychology, 3, 91. https://doi.org/10.3389/feduc.2018.00091

Darby, D., \& Rury, J.L. (2018). The color of mind: Why the origins of the achievement gap matter for justice. The University of Chicago Press. https://doi.org/10.7208/chicago/9780226525495.001.0001

Diener, C.I., \& Dweck, C.S. (1978). An analysis of learned helplessness: Continuous changes in performance, strategy and achievement cognitions following failure. Journal of Personality and Social Psychology, 36, 451-462. https://doi.org/10.1037/0022-3514.36.5.451

DuBois, W. (1904). The souls of black folk (p.5). Digireads.com Publishing.

Dweck, C. (2007). Boosting achievement with messages that motivate. Education Canada, 47(2), 6-10.

Dweck, C., \& Leggett, E. (1988). A social-cognitive approach to motivation and personality. Psychological Review, 95, 256-273. https://doi.org/10.1037/0033-295x.95.2.256

Dweck, C.S. (2010). Even geniuses work hard. Educational Leadership, 68(1), 16-20.

Eccles, J.S., \& Wigfield, A. (2002). Motivational beliefs, values, and goals. Annual Review of Psychology, 53, 109-132. http://dx.doi.org/10.1146/annurev.psych.53.100901.135153

Ertmer, P.A., Ottenbreit-Leftwich, A.T., Sadik, O., Sendurur, E., \& Sendurur, P. (2012). Teacher beliefs and technology integration practices: A critical relationship. Computers \& Education, 59(2), 423 435. https://doi.org/10.1016/j.compedu.2012.02.001

Estrada, M., Burnett, M., Campbell, A.G., Campbell, P.B., Denetclaw, W.F., Gutiérrez, C.G., . . Zavala, M. (2016). Improving Underrepresented Minority Student Persistence in STEM. CBE - Life Sciences Education, 15(3), 1-10. https://doi.org/10.1187/cbe.16-01-0038

Estrada, M., Woodcock, A., Hernandez, P.R., \& Schultz, P.W. (2011). Toward a Model of Social Influence that Explains Minority Student Integration into the Scientific Community. Journal of Educational Psychology, 103(1), 206-222. https://doi.org/10.1037/a0022809 
Flake, J.K., Barron, K.E., Hulleman, C., McCoach, B.D., \& Welsh, M.E. (2015). Measuring cost: The forgotten component of expectancy-value theory. Contemporary Educational Psychology, 41, 232-244. https://doi.org/10.1016/j.cedpsych.2015.03.002

Fordham, S., \& Ogbu, J.U. (1986). Black students' school success: Coping with the burden of "acting white." The Urban Review, 18, 176-206. https://doi.org/10.1007/bf01112192

Frenzel, A.C., Goetz, T., Pekrun, R., \& Watt, H.M.G. (2010). Development of mathematics interest in adolescence: Influences of gender,family and school context. Journal of Research on Adolescence, 20, 507-537. http://dx.doi.org/10.1111/j.1532-7795.2010.00645.x

Handal, B. (2003). Teachers' mathematical beliefs: A review. The Mathematics Educator, 13(2).

Hattie, J. (2012). Visible learning for teachers: Maximizing impact on learning. Abingdon, U.K.: Routledge.

Hofer, B.K., \& Pintrich, P.R. (1997). The development of epistemological theories: Beliefs about knowledge and knowing and their relation to learning. Review of Educational Research, 67(1), 88-140. https://doi.org/10.3102/00346543067001088

Jiang, Y., \& Rosenzweig, E.Q. (2021). Using cost to improve predictions of adolescent students' future choice intentions, avoidance intentions, and course grades in mathematics and English. Learning and Individual Differences, 86. https://doi.org/10.1016/j.lindif.2021.101978

Jonsson, A-C., Beach, D., Korp, H., \& Erlandson, P. (2012). Teachers' implicit theories of intelligence: Influences from different disciplines and scientific theories. European Journal of Teacher Education, 35(4), 387-400. http://doi.org/10.1080/02619768.2012.662636

Ladson-Billings, G. (2013). Critical race theory-What it is not! In M. Lynn \& A. Dixson (Eds.), The handbook of critical race theory in education (pp. 34-47). New York, NY: Routledge. https://doi.org/10.4324/9780203155721-12

Lazarides, R., Buchholz, J., \& Rubach, C. (2018). Teacher enthusiasm and self-efficacy, studentperceived mastery goal orientation, and student motivation in mathematics classrooms. Teaching and Teacher Education, 69, 1-10. https://doi.org/10.1016/j.tate.2017.08.017

Lee, K.J., \& Anderson, J.A. (2014). Who is really interested in mathematics? An investigation of lower secondary students' mathematical role models. In J. Anderson, M. Cavanagh, \& A. Prescott (Eds.), Curriculum in focus: Research guided practice (Proceedings of the 36th Annual Conference of the Mathematics Education Research Group of Australasia, pp. 397-404). Sydney: MERGA

Little, R.J.A., \& Rubin, D.B. (2002). Statistical analysis with missing data. Hoboken, NJ: Wiley. http://doi.org/10.1002/9781119013563

Liu Sun, K. (2018). The Role of Mathematics Teaching in Fostering Student Growth Mindset. Journal for Research in Mathematics Education, 49(3), 330. https://doi.org/10.5951/jresematheduc.49.3.0330

Liu, W.C. (2021). Implicit Theories of Intelligence and Achievement Goals: A Look at Students' Intrinsic Motivation and Achievement in Mathematics. Frontiers in Psychology, 12. https://doi.org/10.3389/fpsyg.2021.593715

Matthews, J.S. (2018). When am I ever going to use this in the real world? Cognitive flexibility and urban adolescents' negotiation of the value of mathematics. Journal of Educational Psychology, 110(5), 726. https://doi.org/10.1037/edu0000242

McGee, E.O. (2020). Black, Brown, Bruised: How Racialized STEM Education Stifles Innovation. Harvard Education Press. https://doi.org/10.1177/15210251211018178

Milner, H.R. (2006). Preservice Teachers' Learning about Cultural and Racial Diversity: Implications for Urban Education. Urban Education, 41(4), 343-375. https://doi.org/10.1177/0042085906289709

Mueller, C.M., \& Dweck, C.S. (1998). Praise for intelligence can undermine children's motivation and performance. Journal of Personality and Social Psychology, 75(1), 33-52. https://doi.org/10.1037/0022-3514.75.1.33

National Science Foundation, National Center for Science and Engineering Statistics. (2015). Women, Minorities, and Persons with Disabilities in Science and Engineering: 2015. Special Report NSF, pp. 15-311. Arlington, VA. Retrieved from http://www.nsf.gov/statistics/wmpd/ 
Nicholls, J.G. (1984). Achievement motivation: Conceptions of ability, subjective experience, task choice, and performance. Psychological Review, 91, 328-346. http://doi.org/10.1037/0033295X.91.3.328

Ostrander, R.R. (2015). School funding: Inequality in district funding and the disparate impact on urban and migrant school children. BYU Educ. \& L.J., 271.

Paunesku, D., Walton, G.M., Romero, C., Smith, E.N., Yeager, D.S., \& Dweck, C.S. (2015). Mindset interventions are a scalable treatment for academic underachievement. Psychological Science, 26(6) 784-793. https://doi.org/10.1177/0956797615571017

Perez, T., Cromley, J.G., \& Kaplan, A. (2014). The role of identity development, values, and costs in college STEM retention. Journal of Educational Psychology, 106(1), 315-329. https://doi.org/10.1037/a0034027

Prawat, R.S. (1992). Teacher's belief about teaching and learning: A constructivist perspective. American Journal of Education, 100(3), 354-395.

Rattan, A., Good, C., \& Dweck, C.S. (2012). "It's ok—Not everyone can be good at math": Instructors with an entity theory comfort (and demotivate) students. Journal of Experimental Social Psychology, 48(3), 731-737. https://doi.org/10.1016/j.jesp.2011.12.012

Robinson, K.A., Lee, Y.K., Bovee, E.A., Perez, T., Walton, S.P., Briedis, D., \& Linnenbrink-Garcia, L. (2019). Motivation in transition: Development and roles of expectancy, task values, and costs in early college engineering. Journal of Educational Psychology, 111(6), 1081. https://doi.org/10.1037/edu0000331

Schmidt, J.A., Shumow, L., \& Kackar-Cam, H.Z. (2017). Does Mindset Intervention Predict Students' Daily Experience in Classrooms? A Comparison of Seventh and Ninth Graders' Trajectories. Journal of Youth and Adolescence, 46(3), 582-602. https://doi.org/10.1007/s10964-016-0489-z

Shalter Bruening, P. (2010). Pre-Service teacher beliefs about student motivation (Doctoral dissertation). Columbus, $\mathrm{OH}$ : The Ohio State University.

Seals, C. (2016). Expectancy Value Theory: Racializing values in motivation theory using racial opportunity cost. Urban Education Research \& Policy Annuals, 4(2), 56-70. Retrieved from https://journals.uncc.edu/urbaned/article/view/597/599

Sherman, D.K., \& Hartson, K.A. (2011). Reconciling self-protection with self-improvement: Selfaffirmation theory. In M.D. Alicke \& C. Sedikides (Eds.), Handbook of self-enhancement and self-protection (pp. 128-151).

Steele, C.M. (1997). A threat in the air: How stereotypes shape intellectual identity and performance. American Psychologist, 52(6), 613-629. https://doi.org/10.1037/0003-066x.52.6.613

Stephens, N.M, Covarrubias, R., Fryberg, S.A., Markus, H.R., \& Johnson, C.S. (2012). Unseen disadvantage: How American universities' focus on independence undermines the academic performance of first-generation college students. Journal of Personality and Social Psychology, 102(6), 1178-1197. https://doi.org/10.1037/a0027143

Stipek, D.J., Givvin, K.B., Salmon, J.M., \& MacGyvers, V.L. (2001). Teachers' beliefs and practices related to mathematics instruction. Teaching and Teacher Education, 17(2), 213-226.

Stovall, D. (2006). We Can Relate: Hip-Hop Culture, Critical Pedagogy, and the Secondary Classroom. Urban Education, 41(6), 585-602. https://doi.org/10.1177/0042085906292513

Trautwein, U., Marsh, H.W., Nagengast, B., Lüdtke, O., Nagy, G., \& Jonkmann, K. (2012). Probing for the multiplicative term in modern expectancy-value theory: A latent interaction modeling study. Journal of Educational Psychology, 104, 763-777. https://doi.org/10.1037/a0027470

U.S. Department of Education, National Center for Education Statistics. (2005). The Condition of Education 2005 (NCES 2005-094). Washington, DC: U.S. Government Printing Office.

United States Census Bureau. (2019). QuickFacts United States. Census.gov. Retrieved from https://www.census.gov/quickfacts/fact/table/US/PST045219

Vassallo, S. (2013). Self-regulated learning: An application of critical educational psychology. New York: Peter Lang. 
Vaught, S.E. (2009). The color of money: School funding and the commodification of black children. Urban Education, 44(5), 545-570. https://doi.org/10.1177/0042085908318776

Vygotsky, L.S. (1981). The genesis of higher mental functions. In J.V. Wertsch (Ed. \& Trans.), The concept of activity in Soviet psychology (pp. 144-188). New York: Sharpe.

Walton, M.G. \& Cohen, L.G. (2011). A Brief Social-Belonging Intervention Improves Academic and Health Outcomes of Minority Students. Science, 331(6023), 1447. https://doi.org/10.1126/science.1198364

Wang, M-T., \& Degol, J. (2013). Motivational pathways to STEM career choices: Using expectancyvalue perspective to understand individual and gender differences in STEM fields. Developmental Review, 33, 304-340. http://doi.org/10.1016/j.dr.2013.08.001

West, B.T., Welch, K.B., \& Galecki, A.T. (2014). Linear mixed models: A practical guide using statistical software. CRC Press.

Wiesman, J. (2012). Student Motivation and the Alignment of Teacher Beliefs. The Clearing House, 85(3), 102-108.

Wigfield, A., \& Eccles, J.S. (2000). Expectancy-value theory of achievement motivation. Contemporary Educational Psychology, 25, 65-81.

Wigfield, A., Rosenzweig, E., \& Eccles, J. (2017). Achievement values. In A.J. Elliot, C.S. Dweck, \& D.S. Yeager (Eds.), Handbook of competence and motivation: Theory and application (pp. 116134). (2nd ed.). New York, NY: Guilford Press.

Wigfield, A., Tonks, S., \& Eccles, J.S. (2004). Expectancy value theory in cross-cultural perspective. Big Theories Revisited, 4, 165.

Williams, T.L. (2020). 'Underrepresented Minority' Considered Harmful, Racist Language. Communications of the ACM. Retrieved from https://cacm.acm.org/blogs/blog-cacm/245710underrepresented-minority-considered-harmful-racist-language/fulltext

Wu, J.T.C. (2017). The Origins of the Model Minority. Journal of American Ethnic History, 36(2), 99101. https://doi.org/10.5406/jamerethnhist.36.2.0099

Yeager, D.S., \& Walton, G.M. (2011). Social-psychological interventions in education: They're not magic. Review of Educational Research, 81(2), 267-301. https://doi.org/10.3102/0034654311405999

Yeskel, F. (2008). Coming to class: Looking at education through the lens of class; Introduction to the Class and Education special issue. Equity \& Excellence in Education, 41(1), 1-11. https://doi.org/10.1080/10665680701793428

Zee, M., \& Koomen, H.M. (2016). Teacher self-efficacy and its effects on classroom processes, student academic adjustment, and teacher wellbeing: A synthesis of 40 years of research. Review of Educational Research, 86(4), 981-1015. https://doi.org/10.3102/0034654315626801

Zeeb, H., Renkl, A., \& Ostertag, J. (2020). Towards a Growth Mindset Culture in the Classroom: Implementation of a Lesson-Integrated Mindset Training. Education Research International. https://doi.org/10.1155/2020/8067619 